\title{
Families with parents and children living with HIV described strategies to meet 3 common goals for normalisation
}

Rehm RS, Franck LS. Long-term goals and normalization strategies of children and families affected by HIV/AIDS. Adv Nurs Sci 2000 Sep;23:69-82.

\section{QUESTION: What are the long term goals and normalisation strategies of families with parents and children living with HIV?}

Design

Interpretive study based on symbolic interaction and using ethnographic data collection methods.

Setting

Urban paediatric referral centre in the US.

\section{Participants}

21 participants: 9 children with HIV infection (age range 7-15 y), 6 mothers and 1 father with HIV infection (mean age 38 y), and 5 uninfected adult family member caregivers (age range 30-79 y). Participants were of diverse ethnicity.

\section{Methods}

Semistructured, open ended interviews were conducted individually for children (20-45 min) and adults (60-120 min), except for 1 mother and son who requested to be interviewed together. The interview guide included questions on family composition, disease disclosure, and symptom management. Interviews were audiotaped, transcribed verbatim, and data were analysed using constant comparison. Repeat interviews with 4 key participants validated and expanded findings.

\section{Main findings}

Families had 3 goals to establish normalcy and stability: staying healthy, facilitating children's participation in school and social activities, and enhancing social and emotional wellbeing of family members. Strategies for staying healthy included lifestyle decisions (eg, balanced diet and exercise) and active participation in treatment regimens. Children related taking medications to staying healthy. To reinforce the necessity of the treatment regimen, parents often took their medication on the same schedule as their children; they also tried to allow children some freedom for normal activities within the necessary regimen. Parents juggled multiple responsibilities to care for family members; some focused on guarding their own health to keep their children healthy. In facilitating children's participation in school and social activities, schools served as markers of normal childhood activity and as resources. Management of stigma was important. All families were selective in disclosing information about infection to those outside the family and controlled their children's social lives in order to preserve their ability to be treated normally. Enhancing [the] social and emotional wellbeing of family members was achieved by having extended family members provide supplemental care for children. This helped parents by reducing their stress and increasing rest time, and children valued the relationships developed with extended family members. Families cited spiritual and religious beliefs and practices as important coping strategies. Psychological counsellors provided formal emotional support, whereas other professionals, such as nurses at the HIV centre, provided informal support. Non-infected children were a source of support and concern for parents and children.

\section{Conclusion}

Families with parents and children living with HIV had 3 normalisation goals to foster normal living and used strategies to minimise stigma.

\section{COMMENTARY}

This indepth qualitative study by Rehm and Franck highlights difficulties faced by 8 families affected by HIV/AIDS when they present themselves and their illness to people they encounter as they go about their lives HIV/AIDS is particularly problematic because it is a family illness that has stigma associated both with the diagnosis and the transmission of the infection.

A strength of this study is the recognition of the different perspectives of child and adult family members. The authors note that although data were saturated around the findings presented, the small sample size may have been a limitation. The racially and ethnically diverse sample limited findings to goals and strategies common across cultural groups and did not allow exploration of unique features of particular cultural subgroups. It is not clear how the potential for parental guilt was managed and so it is difficult to assess whether this influenced the accounts that were given by the parents. For example, they might have sought to justify themselves as good parents if they felt judged. We are not told how interviews were adapted to meet the needs of children, who can be unfamiliar with participating in an open ended interview in which there are no correct answers. Some interviews with children were only 20 minutes long, risking superficiality.

Although implications for practice are not specifically addressed, there are messages for nursing from this study. Families limited the release of information about children's diagnosis, choosing to tell some people but not others. Nurses may be the healthcare professionals best placed to discuss with families the safest way to release information to those who need to know. Participants disclosed beliefs they harboured about eventual cure and the confidence inspired by long term survival. These reports provide a basis for understanding reluctance to use unpleasant treatments, such as the protease inhibitors discussed by the families.

The study was conducted in the context of relationships with health professionals that might themselves be "normalised," including participation in social activities and important family events. Families said that they felt free to discuss health and other concerns with the staff at the HIV centre. Normalised relationships with nurses could provide the most suitable environment for disclosure of information and so assist with care of family members affected by HIV/AIDS.

Peter Callery, RGN, RSCN, PhD Senior Lecturer University of Manchester Manchester, UK 